\title{
On the Directional Dependence of Composite Field Operators *
}

\section{Paul Otterson and Wolfhart ZimmermanN}

Department of Physics, New York University, New York, N. Y.

Received July 26, 1971

Abstract. The Wilson expansion of the field operator product $A_{1}\left(x_{1}\right) A_{2}\left(x_{2}\right)$ may be used to define composite operators which are local with respect to $\frac{1}{2}\left(x_{1}+x_{2}\right)$ and depend in addition on a vector $\eta$ proportional to the distance $x_{1}-x_{2}$. It is proved that the composite operators are polynomials in $\eta$, for fixed $\eta^{2} \neq 0$, and that their dependence on $\eta^{2}$ only involves powers of $\eta^{2}$ and $\lg \eta^{2}$.

\section{Introduction}

The composite operators associated with the formal product $A_{1}(x) A_{2}(x)$ of two fields may be conveniently defined as the operators $C_{j}$ appearing in the Wilson expansion

$$
\begin{gathered}
A_{1}\left(x_{1}\right) A_{2}\left(x_{2}\right)=\sum_{j=1}^{k} f_{j}(\varrho) C_{j}(x, \eta)+P_{k+1}(x, \eta, \varrho), \\
x_{1}=x+\varrho \eta, \quad x_{2}=x-\varrho \eta, \quad \varrho>0,
\end{gathered}
$$

where the coefficients $f_{j}$ satisfy

$$
\lim _{\varrho \rightarrow 0} \frac{f_{j+1}(\varrho)}{f_{j}(\varrho)}=0, \quad \lim _{\varrho \rightarrow 0} \frac{P_{k+1}(x, \eta, \varrho)}{f_{k}(\varrho)}=0 .
$$

In a recent paper [1] the expansion (1.1) was derived from general assumptions, and the operators $C_{j}$ were shown to be local in $x$.

The operators $C_{j}$ depend on the vector $x$ of the center-of-mass point and an additional four vector $\eta$ proportional to the distance of the arguments $x_{1}$ and $x_{2}$. The dependence on $\eta$ is related to the directional dependence of composite field operators. This can be seen by setting

$$
\eta=\frac{\xi}{\sqrt{-\xi^{2}}}, \quad \varrho=\sqrt{-\xi^{2}}
$$

* This work was supported in part by the National Science Foundation Grant No. GP-25609. 
in (1.1). The Wilson expansion then takes the more familiar form

$$
A_{1}(x+\xi) A_{2}(x-\xi)=\sum_{j=1}^{k} f_{j}\left(\sqrt{-\xi^{2}}\right) C_{j}\left(x, \frac{\xi}{\sqrt{-\xi^{2}}}\right)+P_{k+1}
$$

where the composite operators $C_{j}$ depend on $x$ and the direction $\eta=\xi / \sqrt{-\xi^{2}}$ of the difference vector $\xi$.

The purpose of this paper is to completely characterize the $\eta$-dependence of the operators $C_{j}$, using only the Wightman postulates [2] together with the additional assumptions of Ref. [1]. In particular, it will be shown that for fixed $\eta^{2} \neq 0$ the operators $C_{j}$ are polynomials in $\eta$, as one should expect from renormalized perturbation theory. Moreover we find that the dependence on $\eta^{2}$ only involves powers of $\eta^{2}$ and $\lg \eta^{2}$.

In this introduction we shortly sketch the proof of the main theorem for the composite operators which appear in the expansion of $A(x+\varrho \eta)$ $A(x-\varrho \eta)$. The rigorous treatment, as well as the generalization to the product of two different operators, will be given in Section 2-7.

The local operators $C_{j}$ are recursively constructed by [1]

$$
\begin{aligned}
P_{1}(x, \eta, \varrho) & =A(x+\varrho \eta) A(x-\varrho \eta) \\
P_{j}(x, \eta, \varrho) & =f_{j}(\varrho) C_{j}(x, \eta)+P_{j+1}(x, \eta, \varrho) \\
C_{j}(x, \eta) & =\lim _{\varrho \rightarrow 0} \frac{P_{j}(x, \eta, \varrho)}{f_{j}(\varrho)} .
\end{aligned}
$$

For the Fourier transforms

we have

$$
\begin{gathered}
\tilde{P}_{j}(x, u, \varrho)=\frac{1}{(2 \pi)^{2}} \int d \eta e^{i \eta u} P_{j}(x, \eta, \varrho) \\
\tilde{C}_{j}(x, u)=\frac{1}{(2 \pi)^{2}} \int d \eta e^{i \eta u} C_{j}(x, \eta)
\end{gathered}
$$

$$
\begin{aligned}
\tilde{P}_{j}(x, u, \varrho) & =f_{j}(\varrho) \tilde{C}_{j}(x, u)+\tilde{P}_{j+1}(x, u, \varrho) \\
\tilde{C}_{j}(x, u) & =\lim _{\varrho \rightarrow 0} \frac{\tilde{P}_{j}(x, u, \varrho)}{f_{j}(\varrho)} .
\end{aligned}
$$

Let $\Phi_{\varrho}, \Psi_{q}$ be eigenvectors of the energy-momentum operator with eigenvalues $p_{\mu}$ or $q_{\mu}$ respectively. The energy-momentum eigenvalues $r$ of an intermediate state is related to $p, q, u$ by

$$
r=\frac{w}{2 \varrho}, \quad w=u+\varrho(p+q) .
$$


Therefore

$$
\left(\Phi_{p}, \tilde{P}_{1}(x, u, \varrho) \Psi_{q}\right)=0 \quad \text { unless } \quad w^{2} \geqq 0, \quad w_{0} \geqq 0 .
$$

Dividing (1.7) by $f_{1}(\varrho)$ and taking the limit $\varrho \rightarrow 0$ we find

$$
\left(\Phi_{p}, \tilde{C}_{1}(x, u) \Psi_{q}\right)=0 \quad \text { unless } \quad u^{2} \geqq 0, \quad u_{0} \geqq 0 .
$$

Repeated application of this argument to the recursion formulae (1.6) leads to

$$
\left(\Phi_{p}, \tilde{C}_{k}(x, u) \Psi_{q}\right)=0 \quad \text { unless } \quad u^{2} \geqq 0, \quad u_{0} \geqq 0 .
$$

By linear superposition of the vectors $\Phi_{p}$ and $\Psi_{q}$ we obtain

$$
\left(\Phi, \tilde{C}_{k}(x, u) \Psi\right)=0 \quad \text { unless } \quad u^{2} \geqq 0, \quad u_{0} \geqq 0
$$

for arbitrary matrix elements. (For a rigorous formulation see Theorem 2 and Corollary of Section 3.)

The causality condition

implies

$$
\left[A\left(x_{1}\right), A\left(x_{2}\right)\right]=0 \quad \text { if } \quad\left(x_{1}-x_{2}\right)^{2}<0
$$

$$
P_{1}(x,-\eta, \varrho)=P_{1}(x, \eta, \varrho) \text { if } \eta^{2}<0 .
$$

Dividing (1.10) by $f_{1}(\varrho)$ and taking the limit $\varrho \rightarrow 0$ we get

$$
C_{1}(x,-\eta)=C_{1}(x, \eta) \text { if } \eta^{2}<0 .
$$

Using the recursion formulae (1.6) the relation

$$
C_{j}(x,-\eta)=C_{j}(x, \eta) \text { for } \eta^{2}<0
$$

follows by induction. This implies that the matrix element

$$
f(\eta)=\left(\Phi,\left(C_{j}(x, \eta)-C_{j}(x,-\eta)\right) \Psi\right)
$$

vanishes for spacelike $\eta$,

The Fourier transform

$$
f(\eta)=0 \quad \text { if } \quad \eta^{2}<0 .
$$

$$
\tilde{f}(u)=\left(\Phi,\left(\tilde{C}_{j}(x, u)-\tilde{C}_{j}(x,-u)\right) \Psi\right)
$$

vanishes for spacelike $u$

$$
\tilde{f}(u)=0 \quad \text { if } \quad u^{2}<0
$$

as follows from (1.9). Because of (1.13) the Jost-Lehmann-Dyson representation [4] may be used to write

$$
\tilde{f}(u)=\int_{0}^{\infty} d \kappa^{2} \int d u^{\prime} \sigma\left(x, u-u^{\prime}, \kappa^{2}\right) \tilde{\Delta}\left(u^{\prime}, \kappa^{2}\right) .
$$


The spectral function $\sigma\left(x, v, \kappa^{2}\right)$ vanishes unless the hyperboloid $(u-v)^{2}=\kappa^{2}$ lies in the region $u^{2} \geqq 0$ (where $\tilde{f}$ may be different from zero). Hence $\sigma$ is non-vanishing only at the origin $v=0$, and so

$$
\sigma=\sum_{m=1}^{M} a^{\mu_{1} \ldots \mu_{m}}\left(x, \kappa^{2}\right) \partial_{\mu_{1}} \ldots \partial_{\mu_{m}} \delta(v) .
$$

With this result $\tilde{f}$ becomes

$$
\tilde{f}(u)=\sum_{m=1}^{M} \int_{0}^{\infty} d \kappa^{2} a^{\mu_{1} \ldots \mu_{m}}\left(x, \kappa^{2}\right) \partial_{\mu_{1}} \ldots \partial_{\mu_{m}} \tilde{\Delta}\left(u, \kappa^{2}\right) .
$$

For $u_{0}>0$ we have

since

$$
\left(\Phi, \tilde{C}_{j}(x, u) \Psi\right)=\sum_{m=1}^{M} \int_{0}^{\infty} d \kappa^{2} a^{\mu_{1} \ldots \mu_{m}}\left(x, \kappa^{2}\right) \partial_{\mu_{1}} \ldots \partial_{\mu_{m}} \tilde{\Delta}^{+}\left(u \kappa^{2}\right)
$$

$$
\tilde{f}(u)=\left(\Phi, \tilde{C}_{j}(x, u) \Psi\right) \text { if } u_{0}>0 .
$$

For $u_{0}<0$ or $u^{2}<0$ both sides of (1.19) vanish. Hence (1.19) is valid for all $u$ except $u=0$. Therefore

$$
\begin{aligned}
\left(\Phi, \tilde{C}_{j}(x, u) \Psi\right)= & \sum_{m=1}^{M} \int_{0}^{\infty} d \kappa^{2} a^{\mu_{1} \ldots \mu_{m}}\left(x, \kappa^{2}\right) \partial_{\mu_{1}} \ldots \partial_{\mu_{m}} \tilde{\Delta}^{+}\left(u, \kappa^{2}\right) \\
& +\sum_{n=1}^{N} b^{v_{1} \ldots v_{N}}\left(x, \kappa^{2}\right) \partial_{v_{1}} \ldots \partial_{v_{N}} \delta(u) .
\end{aligned}
$$

The Fourier transform of (1.21) with respect to $u$ yields

$\left(\Phi, C_{j}(x, \eta) \Psi\right)=\sum_{r=1}^{R} \eta_{\varrho_{1}} \ldots \eta_{\varrho_{r}} t^{\varrho_{1} \cdots \varrho_{r}}\left(x, \eta^{2}-i \varepsilon \eta_{0}\right), \quad \varepsilon \rightarrow+0$.

This is the statement that the matrix elements of the composite operators $C_{j}$ are polynomials in $\eta$, for fixed $\eta^{2} \neq 0$. It should be noted that the proof just outlined goes through under much weaker conditions. It is not necessary to assume relation (2.15) of Ref. [1] which excludes oscillations for $\varrho \rightarrow 0$. Instead the hypotheses of Ref. [1] may be formulated in reference to a particular sequence $\varrho_{n}$ with

$$
\lim _{n \rightarrow \infty} \varrho_{n}=0 .
$$

The Wilson expansion and the recursive construction of composite operators then hold with respect to this sequence which suffices for the derivation of (1.22). 
In Section $6 \mathrm{~b}$ it will be proved that the degree of the polynomial (1.22) stays bounded when the states $\Phi$ and $\Psi$ are varied. This shows that also the operator $C_{j}$ is a polynomial in $\eta$, for $\eta^{2} \neq 0$ given.

Finally we examine the dependence of $C_{j}$ on $\eta^{2}$. A characteristic property of the composite operators is that they obey simple transformation laws under the scaling transformation

$$
\eta \rightarrow \sigma \eta
$$

In order to obtain the scaling law we write the expansion (1.1) in the equivalent form

$$
\begin{aligned}
A(x+\varrho \eta) A(x-\varrho \eta) & =A\left(x,+\frac{\varrho}{\sigma} \sigma \eta\right) A\left(x,-\frac{\varrho}{\sigma} \sigma \eta\right) \\
& =\sum_{j=1}^{k} f_{j}\left(\frac{\varrho}{\sigma}\right) C_{j}(x, \sigma \eta)+P_{k+1}\left(x, \sigma \eta, \frac{\varrho}{\sigma}\right) .
\end{aligned}
$$

According to the uniqueness theorem the operators $C_{j}(x, \eta)$ and $C_{j}(x, \sigma \eta)$ must be related by a transformation of the form [5]

$$
C_{j}(x, \sigma \eta)=\sum_{j^{\prime}=1}^{j} s_{j j^{\prime}}(\sigma) C_{j^{\prime}}(x, \eta) .
$$

The triangular, real $k \times k$ matrices

$$
s(\sigma)=\left\|s_{j j^{\prime}}(\sigma)\right\| \quad j, j^{\prime}=1, \ldots, k
$$

satisfy the multiplication rule

$$
s(\sigma \tau)=s(\sigma) s(\tau)
$$

as follows from the identity

$$
\sum_{j^{\prime}} s_{j j^{\prime}}(\sigma \tau) C_{j^{\prime}}(x, \eta)=C_{j}(x, \sigma \tau \eta)=\sum_{j^{\prime} l} s_{j l}(\sigma) s_{l j^{\prime}}(\tau) C_{j^{\prime}}(x, \eta)
$$

Hence the matrices $s(\sigma)$ form a $k$-dimensional representation of the multiplicative group of the real numbers. These representations are well known, a normal form is given in Section 5. Here we only indicate the general situation for $k=1,2$. The scaling law of $C_{1}$ is

$$
C_{1}(x, \sigma \eta)=\sigma^{c_{1}} C_{1}(x, \eta)
$$

After a suitable transformation of $C_{1}, C_{2}$ (by a triangular $2 \times 2$ matrix) the scaling law of $C_{2}$ becomes either

or

$$
C_{2}(x, \sigma \eta)=\sigma^{c_{1}}\left(\lg \sigma C_{1}(x, \eta)+C_{2}(x, \eta)\right)
$$

$$
C_{2}(x \sigma \eta)=\sigma^{c_{2}} C_{2}(x \eta)
$$


In case of relation (1.27) $C_{2}$ can be written as a linear combination of $C_{1}$ and another operator $Q$ which satisfies a power scaling law

$$
C_{2}(x, \eta)=\lg \sqrt{-\eta^{2}+i \varepsilon \eta_{0}} C_{1}(x, \eta)+Q(x, \eta), \quad \varepsilon \rightarrow+0 .
$$

The operator $Q$ as defined by (1.29) indeed satisfies

We further have

$$
Q(x, \sigma \eta)=\sigma^{c_{1}} Q(x, \eta) \text {. }
$$

and

$$
Q(x,-\eta)=-Q(x, \eta) \quad \text { if } \quad \eta^{2}<0
$$

$$
\tilde{Q}(x, u)=0 \quad \text { unless } \quad u^{2} \geqq 0, \quad u_{0} \geqq 0
$$

where $\tilde{Q}$ denotes the Fourier transform with respect to $\eta$. (1.32) follows from (1.9) and the corresponding property of the Fourier transform of $\lg \sqrt{-\eta^{2}+i \varepsilon \eta_{0}}$.

It will be shown in general (Section 5) that after a suitable equivalence transformation the $C_{j}$ are of the form

$$
C_{j}(x, \eta)=\sum_{n=0}^{N}\left(\lg \sqrt{-\eta^{2}+i \varepsilon \eta_{0}}\right)^{n} Q_{j}^{(n)}(x, \eta) \quad \varepsilon \rightarrow+0
$$

where the operators $Q_{j}^{(n)}$ satisfy a power scaling law

and the conditions

$$
Q_{j}^{(n)}(x, \sigma \eta)=\sigma^{c} Q_{j}^{(n)}(x, \eta)
$$

$$
\begin{aligned}
Q_{j}^{(n)}(x,-\eta) & =Q_{j}^{(n)}(x, \eta) \quad \text { if } \quad \eta^{2}<0 \\
\tilde{Q}_{j}^{(n)}(x, u) & =0 \quad \text { unless } \quad u^{2} \geqq 0, \quad u_{0} \geqq 0 .
\end{aligned}
$$

As in the case of the operators $C_{j}$ the conditions (1.35) imply that the $Q_{j}^{(n)}$ are polynomials in $\eta$, for fixed $\eta^{2} \neq 0$.

$$
Q_{j}^{(n)}(x, \eta)=\sum_{r=1}^{R} \eta_{\varrho_{1}} \ldots \eta_{\ell_{r}} T^{\varrho_{1} \ldots \varrho_{r}}\left(x, \eta^{2}-i \varepsilon \eta_{0}\right) .
$$

Due to the scaling law (1.34) the $T^{e_{1} \ldots e_{r}}$ must be homogeneous in $\sqrt{-\eta^{2}}$ of degree $c-r$. Therefore

$$
Q_{j}^{(n)}(x, \eta)=\left(\sqrt{-\eta^{2}+i \varepsilon \eta_{0}}\right)^{c} \Pi_{j}^{(n)}(x, \zeta) \quad \varepsilon \rightarrow+0
$$

where $\Pi_{j}^{(n)}$ is a polynomial in the components of

$$
\zeta=\frac{\eta}{\sqrt{-\eta^{2}+i \varepsilon \eta_{0}}}
$$

(1.33) and (1.37) state the final result that the $\eta^{2}$-dependence of the composite operators only involves powers of $\eta^{2}$ and $\lg \eta^{2}$. 
The following sections contain a detailed and rigorous derivation of this result, generalized to the product of two different fields. Causality is used in Section 2 to show that the same functions $f_{j}$ may be used in expanding $A_{1} A_{2}$ and $A_{2} A_{1}$. This leads to a locality relation of the composite operators for spacelike $\eta$. Analytic properties of the composite operators in $\eta$ are derived in Section 4 which follow from the support properties of the Fourier transformations (Section 3). After a discussion of the scaling law (Section 5) the $\eta$-dependence is derived in Section 6 by an alternative method which does not make use of the Jost-LehmannDyson representation.

\section{Locality}

The general assumption and notations of Ref. [1] will be used throughout the work that follows. $A_{1}$ and $A_{2}$ denote linear combinations of the basic fields $O_{1}, \ldots, O_{c}$. In addition to Wightman's postulates Hypothesis 3 of Ref. [1] will be assumed which implies that the operator product $A_{1} A_{2}$ of two local field operators $A_{1}, A_{2}$ has the Wilson expansion

$A_{1}(x+\varrho \eta) A_{2}(x-\varrho \eta)=\sum_{j=1}^{k} f_{j}(\varrho) C_{j}^{12}(x, \eta)+P_{k+1}^{12}(x, \eta, \varrho)$ in $\mathscr{S}_{x \eta}^{\prime}\left(D_{0}\right)$.

The functions $f_{j}$ may be chosen to be real, they satisfy

$$
\begin{aligned}
\lim _{\varrho \rightarrow 0} \frac{f_{j+1}(\varrho)}{f_{j}(\varrho)} & =0 \\
\lim _{\varrho \rightarrow 0} \frac{\left(\Phi, P_{k+1}^{12}(x, \eta, \varrho) \Psi\right)}{f_{k}(\varrho)} & =0 \text { in } \mathscr{S}_{x \eta}^{\prime} \text { for } \Phi, \Psi \in D_{0} .
\end{aligned}
$$

The operators $C_{j}^{12}(x, \eta)$ are local in $x$ for given $\eta$. Another consequence of locality can be derived from

valid in

$$
A_{1}(x+\varrho \eta) A_{2}(x-\varrho \eta)= \pm A_{2}(x-\varrho \eta) A_{1}(x+\varrho \eta)
$$

$$
\mathscr{S}_{x}^{\prime}\left(D_{0}\right) \text { for } \eta^{2}<0
$$

To this end we compare (2.1) with the expansion of $A_{2} A_{1}$

$$
A_{2}(x+\varrho \eta) A_{1}(x-\varrho \eta)=\sum_{j=1}^{k} f_{j}^{\prime}(\varrho) C_{j}^{\prime 21}(x, r)+P_{k+1}^{\prime 21}(x, \eta, \varrho) \text { in } \mathscr{S}_{x \eta}^{\prime}\left(D_{0}\right)
$$


For spacelike $\eta$ (2.1) and (2.3) yield

$$
\begin{gathered}
A_{2}(x+\varrho \eta) A_{1}(x-\varrho \eta)=\sum_{j=1}^{k} f_{j}(\varrho)( \pm 1) C_{j}^{12}(x,-\eta) \pm P_{k+1}^{12}(x,-\eta, \varrho) \\
\text { in } \quad \mathscr{S}_{x \eta}^{\prime}\left(D_{0}\right), \quad \eta^{2}<0
\end{gathered}
$$

which is equivalent to (2.4). According to the uniqueness theorem there must be an equivalence transformation

$$
\begin{aligned}
& \pm C_{j}^{12}(x,-\eta)=\sum_{j^{\prime}=1}^{j} a_{j j^{\prime}} C_{j}^{\prime 21}(x, \eta) \\
& \pm P_{k+1}^{12}(x,-\eta, \varrho)=P_{k+1}^{\prime 21}(x, \eta, \varrho)+\sum_{j=1}^{k} h_{j}(\varrho) C_{j}^{\prime 21}(x, \eta) \\
& a_{j j} \neq 0, \quad \lim _{\varrho \rightarrow 0} \frac{h_{j}(\varrho)}{f_{j}(\varrho)}=0
\end{aligned}
$$

valid in $\mathscr{S}_{x \eta}^{\prime}\left(D_{0}\right)$ for $\eta^{2}<0$. The functions $f_{j}$ and $f_{j}^{\prime}$ are related by the asymptotic expansion

$$
f_{j}(\varrho)=\sum_{j^{\prime}=j}^{\infty} a_{j^{\prime} j} f_{j^{\prime}}^{\prime}(\varrho)+h_{j}(\varrho) .
$$

An equivalence transformation for (2.4) which is valid for all $\eta$ may be set up by using (2.7) and defining

$$
\begin{aligned}
& C_{j}^{21}(x, \eta)=\sum_{j^{\prime}=1}^{j} a_{j j^{\prime}} C_{j}^{\prime 21}(x, \eta) \\
& P_{k+1}^{21}(x, \eta, \varrho)=P_{k+1}^{21}(x, \eta, \varrho)+\sum_{j=1}^{k} k_{j}(\varrho) C_{j}^{\prime 21}(x, \eta) \\
& \text { in } \mathscr{S}_{x \eta}^{\prime}\left(D_{0}\right) .
\end{aligned}
$$

Applying (2.7-2.8) to (2.4) we find

$$
A_{2}(x+\varrho \eta) A_{1}(x-\varrho \eta)=\sum_{j=1}^{k} f_{j}(\varrho) C_{j}^{21}(x, \eta)+P_{k}^{21}(x, \eta \varrho) \text { in } \mathscr{S}_{x \eta}^{\prime}\left(D_{0}\right)
$$

as an equivalent form of (2.4). We thus have

Theorem 1. As a consequence of causality the operator products $A_{1} A_{2}$ and $A_{2} A_{1}$ may be expanded with the same coefficients $f_{j}$

$$
\begin{gathered}
A_{a}(x+\varrho \eta) A_{b}(x-\varrho \eta)=\sum_{j=1}^{k} f_{j}(\varrho) C_{j}^{a b}(x, \eta)+P_{k+1}^{a b}(x, \eta \varrho) \text { in } \mathscr{S}_{x \eta}^{\prime}\left(D_{0}\right) \\
(a, b=1,2) .
\end{gathered}
$$


For spacelike $\eta$ the operators $C_{j}^{12}$ and $C_{j}^{21}$ are related by

$$
C_{j}^{21}(x,-\eta)= \pm C_{j}^{12}(x, \eta) \text { in } \mathscr{S}_{x \eta}^{\prime}, \quad \eta^{2}<0 .
$$

\section{Support Properties}

We begin with the derivation of some support properties for the composite operator $C_{1}$. For the time being we consider matrix elements of $A_{a}(x+\varrho \eta) A_{b}(x-\varrho \eta)$ and $C_{1}^{a b}(x \eta)$ between vectors $\Phi, \Psi \in B . B$ denotes the domain of all vectors which can be obtained by applying polynomials of the basic fields $O_{j}(f)$ to the vacuum where the Fourier transform of the test functions $f$ has compact support. Since such vectors are of bounded energy-momentum it will be possible to establish support properties in momentum space. It is sufficient to take vectors of the special form

$$
\begin{aligned}
& \Phi=A_{1}^{\prime}\left(f_{1}\right) \ldots A_{n}^{\prime}\left(f_{n}\right) \Omega \in B \\
& \Psi=A_{1}^{\prime \prime}\left(g_{1}\right) \ldots A_{m}^{\prime \prime}\left(g_{m}\right) \Omega \in B
\end{aligned}
$$

where $A_{j}^{\prime}, A_{j}^{\prime \prime}$ denote basic fields $O_{1}, \ldots, O_{c}$. We first express

$$
\phi^{a b}(x, \eta \varrho)=\left(\Phi, A_{a}(x+\varrho \eta) A_{b}(x-\varrho \eta) \Psi\right) \quad a, b=1,2
$$

by a Wightman function in momentum space. To this end we form the Fourier integral

$$
\begin{aligned}
\phi^{a b}(x, \eta, \varrho) & =\frac{1}{(2 \pi)^{4}} \int d r_{1} d r_{2} e^{-i x\left(r_{1}+r_{2}\right)-i \varrho \eta\left(r_{1}-r_{2}\right)}\left(\Phi, \tilde{A}_{a}\left(r_{1}\right) \tilde{A}_{b}\left(r_{2}\right) \Psi\right) \\
& =\frac{1}{(4 \pi \varrho)^{4}} \int d u d v e^{-i \eta u-i x v}\left(\Phi, \tilde{A}_{a}\left(r_{1}\right) \tilde{A}_{b}\left(r_{2}\right) \Psi\right)
\end{aligned}
$$

with

$$
\begin{aligned}
v & =r_{1}+r_{2}, \quad u=\varrho\left(r_{1}-r_{2}\right) \\
r_{1} & =\frac{v}{2}+\frac{u}{2 \varrho}, \quad r_{2}=\frac{v}{2}-\frac{u}{2 \varrho} .
\end{aligned}
$$

The Fourier transform of $\phi^{a b}$ with respect to $\eta$ becomes

$$
\begin{aligned}
\tilde{\phi}^{a b}(x, u, \varrho) & =\frac{1}{(2 \pi)^{2}} \int d \eta e^{i \eta u} \phi^{a b}(x, \eta \varrho) \\
& =\frac{1}{(2 \varrho)^{4}(2 \pi)^{2}} \int d v e^{-i x v}\left(\Phi, \tilde{A}_{a}\left(r_{1}\right) \tilde{A}_{b}\left(r_{2}\right) \Psi\right)
\end{aligned}
$$


inserting the state vectors (3.1) we obtain

$$
\begin{aligned}
\tilde{\phi}^{a b}(x, u, \varrho)= & \frac{1}{(2 \varrho)^{4}(2 \pi)^{2}} \int d p d q e^{-i x(Q-P)} \tilde{f}_{1}\left(p_{1}\right) \ldots \tilde{f}_{n}\left(p_{n}\right) \tilde{g}_{1}\left(q_{1}\right) \ldots \tilde{g}_{m}\left(q_{m}\right) \\
& \cdot W\left(S_{1}, \ldots, S_{n+m+1}\right)
\end{aligned}
$$

with

$$
\begin{aligned}
& d p=d p_{1} \ldots d p_{n}, \quad d q=d q_{1} \ldots d q_{m}, \quad P=-\sum_{j=1}^{n} p_{j}, \quad Q=\sum_{j=1}^{m} q_{j} \\
& S_{1}=p_{n}, \quad S_{2}=-p_{n}-p_{n-1}, \ldots, S_{n}=P \\
& S_{n+1}=\frac{P+Q}{2}+\frac{u}{2 \varrho} \\
& S_{n+2}=Q, \quad S_{n+3}=q_{2}-\cdots-q_{m}, \ldots, S_{n+m+1}=-q_{1} .
\end{aligned}
$$

The Wightman function $W$ is defined by

$$
\begin{gathered}
\left\langle\tilde{A}_{n}^{\prime *}\left(-p_{n}\right) \ldots \tilde{A}_{1}^{\prime *}\left(-p_{1}\right) \tilde{A_{a}}\left(r_{1}\right) \tilde{A_{b}}\left(r_{2}\right) \tilde{A}_{1}^{\prime \prime}\left(q_{1}\right) \ldots A_{m}^{\prime \prime}\left(q_{m}\right)\right\rangle \\
=\delta(P+v-Q) W\left(S_{1}, \ldots, S_{n+m+1}\right)
\end{gathered}
$$

where translation invariance was used for separating $\delta(P+v-Q)$ [6]. $\tilde{A}_{j}^{\prime *}$ denotes the Fourier transform of the adjoint $A_{j}^{*}(x)$, i.e.,

$$
A_{j}^{* *}\left(-p_{j}\right)=\tilde{A}_{j}^{\prime}\left(p_{j}\right)^{*} .
$$

According to (3.5-3.7), $\tilde{\phi}$ is a tempered distribution in $u$ for given $x$. Hence $\phi$ is a tempered distribution in $\eta$. In a similar way translation invariance can be used to show that

$$
g^{a b}(x, \eta)=\left(\Phi, C_{1}^{a b}(x, \eta) \Psi\right) \quad \Phi, \Psi \in B
$$

is a tempered distribution in $\eta$ only. The relation

$$
\left(\Phi, C_{1}^{a b}(x, \eta) \Psi\right)=\lim _{\varrho \rightarrow 0} \frac{\left(\Phi, A_{a}(x+\varrho \eta) A_{b}(x-\varrho \eta) \Psi\right)}{f_{1}(\varrho)} \text { in } \mathscr{S}_{x \eta}^{\prime} \text { for } \Phi, \Psi \in B
$$

may therefore be interpreted as a limit relation of tempered distributions in $\eta$ at any value of $x$

$$
g^{a b}(x, \eta)=\lim _{\varrho \rightarrow 0} \frac{\phi^{a b}(x, \eta \varrho)}{f_{1}(\varrho)} \text { in } \mathscr{S}_{\eta}^{\prime} .
$$

For the Fourier transforms $\tilde{\phi}$ and

$$
\tilde{g}^{a b}(x, u)=\frac{1}{(2 \pi)^{2}} \int d \eta e^{i \eta u} g^{a b}(x, \eta)
$$


we obtain the relation

$$
\tilde{g}^{a b}(x, u)=\lim _{\varrho \rightarrow 0} \frac{\tilde{\Phi}^{a b}(x, u, \varrho)}{f_{1}(\varrho)} \text { in } \mathscr{S}_{u}^{\prime} .
$$

The support properties of $\tilde{\phi}$ follow from the support properties of the Wightman function (3.7). We obtain

unless

$$
\tilde{\phi}^{a b}(x, u, \varrho)=0
$$

for at least one vector

$$
u+\varrho(P+Q) \in \bar{V}_{+}
$$

$$
P+Q \in C
$$

The compact set $C$ is given by the conditions

$$
\begin{array}{ll}
P=-\Sigma p_{j}, & Q=+\Sigma q_{j} \\
p_{j} \in \operatorname{supp} \tilde{f}_{j}, & q_{j} \in \operatorname{supp} \tilde{q}_{j} \\
-p_{1} \in \bar{V}_{+}, & -p_{1}-p_{2} \in \bar{V}_{+}, \ldots, P \in \bar{V}_{+} \\
-q_{1} \in \bar{V}_{+}, & -q_{1}-q_{2} \in \bar{V}_{+}, \ldots, Q \in \bar{V}_{+}
\end{array}
$$

(3.13-3.17) then implies that

$$
\tilde{g}^{a b}(x, u)=0 \quad \text { unless } u \in \bar{V}_{+} .
$$

For if $u \notin \bar{V}_{+}$we can find a value $\varepsilon>0$ such that

$$
u+\varrho(P+Q) \notin \bar{V}_{+} \quad \text { for any } P+Q \in C
$$

provided $\varrho \leqq \varepsilon$.

The result (3.18) can easily be carried over to the general case of the operator $C_{j}^{a b}$. To this end we form matrix elements of $P_{j}^{a b}$ and $C_{j}^{a b}$ between vectors $\Phi, \Psi$ of the form (3.1)

$$
\begin{aligned}
\phi_{j}^{a b}(x, \eta \varrho) & =\left(\Phi, P_{j}^{a b}(x, \eta \varrho) \Psi\right) \\
g_{j}^{a b}(x, \eta) & =\left(\Phi, C_{j}^{a b}(x, \eta) \Psi\right) \\
\tilde{\phi}_{j}^{a b}(x, u, \varrho) & =\frac{1}{(2 \pi)^{2}} \int d \eta e^{i \eta u} \phi_{j}^{a b}(x, \eta \varrho) \\
\tilde{g}_{j}^{a b}(x, u) & =\frac{1}{(2 \pi)^{2}} \int d \eta e^{i \eta u} g_{j}^{a b}(x, \eta) .
\end{aligned}
$$

Using the recursion formulae

$$
\begin{aligned}
\tilde{\phi}_{j}^{a b}(x, u, \varrho) & =f_{j}(\varrho) \tilde{g}_{j}^{a b}(x, u)+\tilde{\phi}_{j+1}^{a b}(x, u, \varrho) \\
\tilde{g}_{j}^{a b}(x, u) & =\lim _{\varrho \rightarrow 0} \frac{\tilde{\phi}_{j}^{a b}(x, u, \varrho)}{f_{j}(\varrho)}
\end{aligned}
$$


we obtain by induction that $\tilde{\phi}_{j}^{a b}, \tilde{g}_{j}^{a b}$ are distributions in $\mathscr{S}_{u}^{\prime}$ with

and

$$
\tilde{g}_{j}^{a b}(x, u)=0 \quad \text { unless } u \in \bar{V}_{+}
$$

$$
\tilde{\phi}_{j}^{a b}(x, u, \varrho)=0
$$

unless $u$ satisfies (3.15-3.17) or $u \in \bar{V}_{+}$. We summarize the results by the following

Theorem 2. For given $x$ and vectors $\Phi, \Psi \in B$ the matrix element

$$
g_{j}^{a b}(x, \eta)=\left(\Phi, C_{j}^{a b}(x, \eta) \Psi\right) \quad a, b=1,2
$$

is a tempered distribution in $\eta$. Its Fourier transform with respect to $\eta$ vanishes unless $u^{2} \geqq 0, u_{0} \geqq 0$.

Using continuity in $\Phi$ and $\Psi$ we obtain the following

Corollary. The Fourier transform $\tilde{g}_{k}^{a b}(x, u)$ of

$$
\begin{gathered}
g_{k}^{a b}(x, \eta)=\left(\Phi, C_{k}^{a b}(x, \eta) \Psi\right) \in \mathscr{S}_{x}^{\prime} \\
a, b=1,2, \quad \Phi \in H, \Psi \in D_{0}
\end{gathered}
$$

with respect to $\eta$ vanishes unless $u^{2} \geqq 0, u_{0} \geqq 0$.

Proof. For $\Phi \in B$ given, (3.24) is continuous in $\Psi$. Hence the statement follows for all vectors $\Phi \in B, \Psi \in H$. Keeping now $\Psi \in D_{0}$ fixed and using continuity in $\Phi$ the theorem follows.

\section{Analyticity}

In this section we establish some analytic properties of the composite operators in the variable $\eta$. We first introduce linear combinations of $C_{j}^{12}, C_{j}^{21}$ which are even or odd respectively for spacelike $\eta$

$$
\begin{aligned}
C_{j}^{\text {even }}(x, \eta) & =C_{j}^{12}(x, \eta) \pm C_{j}^{21}(x, \eta) \\
C_{j}^{\text {odd }}(x, \eta) & =C_{j}^{12}(x, \eta) \mp C_{j}^{21}(x, \eta) .
\end{aligned}
$$

The signs are chosen corresponding to the signs in Eq. (2.9) and (2.10). Matrix elements between vectors (3.1) are denoted by

$$
\begin{aligned}
g_{j}^{\text {even }}(x, \eta) & =\left(\Phi, C_{j}^{\text {even }}(x, \eta) \Psi\right)=g_{j}^{12}(x, \eta) \pm g_{j}^{21}(x, \eta) \\
g_{j}^{\text {odd }}(x, \eta) & =\left(\Phi, C_{j}^{\text {odd }}(x, \eta) \Psi\right)=g_{j}^{12}(x, \eta) \mp g_{j}^{21}(x, \eta) .
\end{aligned}
$$

For spacelike $\eta$

$$
\begin{aligned}
C_{j}^{\text {even }}(x,-\eta) & =C_{j}^{\text {even }}(x, \eta), \quad g_{j}^{\text {even }}(x,-\eta)=g_{j}^{\text {even }}(x, \eta) \\
C_{j}^{\text {odd }}(x,-\eta) & =-C_{j}^{\text {odd }}(x, \eta), \quad g^{\text {odd }}(x,-\eta)=-g_{j}^{\text {odd }}(x, \eta) \text { if } \quad \eta^{2}<0 .
\end{aligned}
$$


$g_{j}^{\text {even }}$ and $g_{j}^{\text {odd }}$ have the support properties of Theorem 2 which imply the following theorem on the analytic continuation of $g_{j}^{()}(x, \eta)$ in $\eta$ (see for instance Ref. 2, Section 2-3). (' stands for the superscript even or odd.

Theorem 3. The matrix element

$$
g_{j}^{()}(x, \eta)=\left(\Phi, C_{j}^{()}(x, \eta) \Psi\right) \quad \Phi, \Psi \in B
$$

is the boundary value

$$
g_{j}^{()}\left(x, \eta_{1}\right)=\lim _{\eta_{2} \rightarrow+0} G_{j}^{()}\left(x, \eta_{1}-i \eta_{2}\right) \text { in } \mathscr{S}_{\eta_{1}}^{\prime}
$$

of an analytic function $G_{j}^{()}(x, \eta)$ which is regular and bounded by a polynomial inside the cone $-\operatorname{Im} \eta \in V_{+}$.

The functions

$$
\hat{G}_{j}^{\text {even }}(x, \eta)=G_{j}^{\text {even }}(x,-\eta) ; \quad \hat{G}_{j}^{\text {odd }}(x, \eta)=-G_{j}^{\text {odd }}(x,-\eta)
$$

are analytic and bounded by a polynomial inside the cone $\operatorname{Im} \eta \in V_{+}$.

$$
\begin{aligned}
\lim _{-\eta_{2} \rightarrow+0} \hat{G}_{j}^{()}\left(x, \eta_{1}-i \eta_{2}\right) & = \pm g_{j}^{()}\left(x,-\eta_{1}\right) \\
& =g_{j}^{()}\left(x, \eta_{1}\right) \text { for } \eta_{1}^{2}<0 .
\end{aligned}
$$

$G_{j}^{()}(x, \eta)$ and $\hat{G}_{j}^{()}(x, \eta)$ are analytic at spacelike $\eta$ and continuations of each other

$$
G_{j}^{()}(x, \eta)=\hat{G}_{j}^{()}(x, \eta) \text { for } \eta \text { real and } \eta^{2}<0 .
$$

Remark. In the preceding theorem and the work that follows an analytic function $F(\eta)$ which is regular in $\mp \operatorname{Im} \eta \in V_{+}$is called bounded by a polynomial inside this region if there exists a polynomial $P_{u}(\eta)$ depending on a four vector $u \in V_{+}$such that

$$
|F(\eta)| \leqq\left|P_{u}(\eta)\right|
$$

for any $\operatorname{Re} \eta$ and $\mp \operatorname{Im} \eta=u+v$ with $u \in V_{+}$fixed, $v \in V_{+}$arbitrary.

As a consequence of the Corollary to Theorem 2 we have

Theorem 4. Let $w(\eta)$ be a function which is the boundary value

$$
w\left(\eta_{1}\right)=\lim _{\eta_{2} \rightarrow+0} W\left(\eta_{1}-i \eta_{2}\right)
$$

of an analytic function $W(\eta)$, regular and bounded by a polynomial inside the cone $-\operatorname{Im} \eta \in V_{+}$. Then

defines an operator in $\mathscr{S}_{x \eta}^{\prime}\left(D_{0}\right)$.

$$
w(\eta) C_{j}^{a b}(x, \eta)
$$

9 Commun. math. Phys., Vol. 24 
Proof. The matrix element

$$
m(\eta)=\int d x s(x)\left(\Phi, C_{k}^{a b}(x, \eta) \Psi\right) ; \quad \Phi \in H, \Psi \in D_{0}, s \in \mathscr{S}\left(R_{4}\right)
$$

is the boundary value

$$
m\left(\eta_{1}\right)=\lim _{\eta_{2} \rightarrow+0} M\left(\eta_{1}-i \eta_{2}\right)
$$

of an analytic function $M(\eta)$ which is regular and bounded by a polynomial inside the cone $-\operatorname{Im} \eta \in V_{+}$. Therefore the product $w(\eta) m(\eta)$ is well defined by

$$
w\left(\eta_{1}\right) m\left(\eta_{1}\right)=\lim _{\eta_{2} \rightarrow+0} W\left(\eta_{1}-i \eta_{2}\right) M\left(\eta_{1}-i \eta_{2}\right) .
$$

Since (4.7) is linear and continuous in $\Phi$ there exists a vector $\hat{\Psi}$ with

The equation

$$
\int d \eta u(\eta) W(\eta) m(\eta)=(\Phi, \hat{\Psi}) .
$$

$$
\int d x d \eta s(x) u(\eta) C_{k}^{a b}(x, \eta) \Psi=\hat{\Psi} \quad u \in \mathscr{S}\left(R_{4}\right)
$$

defines a linear operator in $\mathscr{S}_{x \eta}^{\prime}\left(D_{0}\right)$.

\section{Scaling Law}

We will make use of the fact that

$$
P^{a b}(x, \eta, \varrho)=A_{a}(x+\varrho \eta) A_{b}(x-\varrho \eta)
$$

depends on $\varrho$ and $\eta$ through the product $\varrho \eta$ only. For every test function $t \in \mathscr{S}_{x \eta}$ we have

$$
P^{a b}(t, \varrho)=P^{a b}\left(t_{\sigma}, \frac{\varrho}{\sigma}\right) \text { on } D_{0}
$$

where $t_{\sigma}$ is the test function

(5.1) implies

$$
t_{\sigma}(x, \eta)=\sigma^{-4} t\left(x, \frac{\eta}{\sigma}\right) .
$$

with

$$
P^{a b}(t, \varrho)=\sum_{j=1}^{k} f_{j}\left(\frac{\varrho}{\sigma}\right) C_{\sigma j}^{a b}(t)+P_{k+1}^{a b}\left(t_{\sigma}, \frac{\varrho}{\sigma}\right)
$$

$$
C_{\sigma j}^{a b}(t)=C_{j}^{a b}\left(t_{\sigma}\right) .
$$


Since (5.3) is an equivalent form of the Wilson expansion the uniqueness theorem implies the scaling law

or

$$
C_{\sigma j}^{a b}(t)=\sum_{j^{\prime}=1}^{j} s_{j j^{\prime}}(\sigma) C_{j^{\prime}}^{a b}(t)
$$

$$
C_{j}^{a b}(\sigma \eta)=\sum_{j^{\prime}=1}^{j} s_{j j^{\prime}}(\sigma) C_{j^{\prime}}^{a b}(\eta)
$$

with real coefficients $s_{j j^{\prime}}$. Since the matrix elements of $C_{j}^{a b}$ between vectors $\Phi, \Psi \in B$ are analytic for spacelike $\eta$ the functions $s_{j j^{\prime}}$ must be differentiable.

For the moment we restrict ourselves to the first $k$ composite operators. We write the first $k$ equations of (5.6) in matrix form

with

$$
C^{a b}(\sigma \eta)=s(\sigma) C^{a b}(\eta)
$$

$$
s=\left(\begin{array}{ccccc}
s_{11} & 0 & 0 & \ldots & 0 \\
s_{21} & s_{22} & 0 & \ldots & 0 \\
\cdot & \cdot & \cdot & & \cdot \\
\cdot & \cdot & \cdot & & \cdot \\
\cdot & \cdot & \cdot & & \cdot \\
s_{k 1} & s_{k 2} & s_{k 3} & \ldots & s_{k k}
\end{array}\right), \quad C^{a b}=\left(\begin{array}{c}
C_{1}^{a b} \\
C_{2}^{a b} \\
\cdot \\
\cdot \\
\cdot \\
C_{k}^{a b}
\end{array}\right) \text {. }
$$

The multiplication law

$$
s(\sigma \tau)=s(\sigma) s(\tau)
$$

follows from the identity

$$
s(\sigma \tau) C(\eta)=C(\sigma \tau \eta)=s(\sigma) s(\tau) C(\eta) .
$$

Accordingly $s(\sigma)$ is a $k$-dimensional, real and differentiable representation of the multiplicative group of the real numbers. Introducing new composite operators

$$
\begin{gathered}
C^{\prime}=T C \quad T_{j j^{\prime}}=0 \quad \text { for } \quad j<j^{\prime} \\
C^{\prime}(\sigma \eta)=s^{\prime}(\sigma) C^{\prime}(\eta)
\end{gathered}
$$

by a suitable triangular matrix $T$ the matrices $s$ can be transformed into [7]

$$
s^{\prime}=\left(\begin{array}{c|ccc}
s_{1} & 0 & & \\
\hline 0 & s_{2} & & \\
\hline 0 & 0 & \ddots & 0 \\
\hline & & 0 & s_{n}
\end{array}\right)
$$


where each $s_{j}$ is an $N_{j} \times N_{j}$ matrix of the normal form

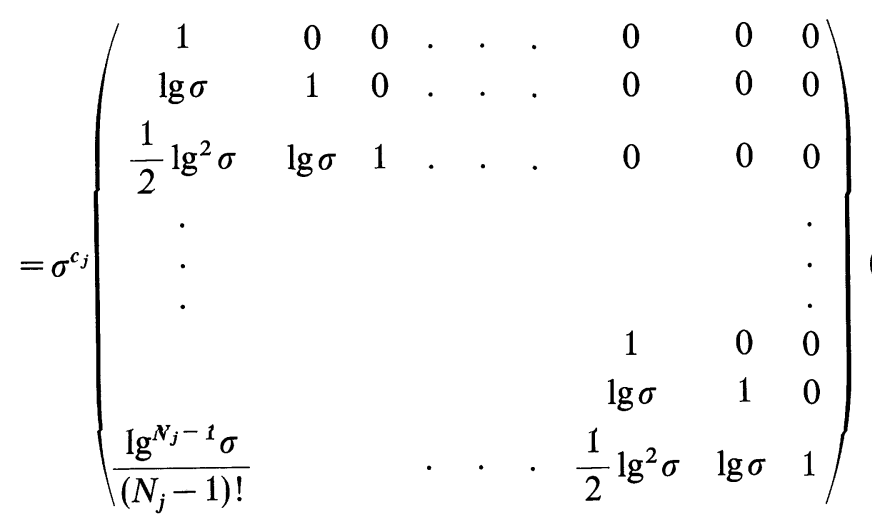

with real exponents $c_{j}$.

It is convenient to relabel the operators $C_{j}^{\prime}$ according to the decomposition of $s^{\prime}$ into submatrices $s_{j}$

$$
C_{11}, \ldots, C_{1 N_{1}} ; \quad C_{21} \ldots \quad ; \quad C_{n 1}, \ldots, C_{n N_{n}}
$$

(5.17) may then be broken up into relations

with

$$
C_{(j)}(\sigma \eta)=s_{j}(\sigma) C_{(j)}(\eta)
$$

$$
C_{(j)}=\left(\begin{array}{c}
C_{j 1} \\
\vdots \\
C_{j N_{j}}
\end{array}\right) .
$$

The scaling law (5.14) can be used to continue the matrix element

$$
g_{j k}^{a b}(x, \eta)=\left(\Phi, C_{j k}^{a b}(x, \eta) \Psi\right) \quad \Phi, \Psi \in B
$$

beyond the domain given in Theorem 3 . Since both sides of

$$
g_{j l}^{a b}(x, \sigma \eta)=\sum_{l^{\prime}=1}^{l} s_{j l l^{\prime}}(\sigma) g_{j l^{\prime}}^{a b}(x, \eta)
$$

are boundary values of analytic functions in $\eta$ a similar relation holds for their continuations

$$
G_{j l}^{a b}(x, \sigma \eta)=\sum_{l^{\prime}=1}^{l} s_{j l l^{\prime}}(\sigma) G_{j l^{\prime}}^{a b}(x, \eta) \quad \text { if } \quad-\operatorname{Im} \eta \in V_{+} .
$$

For given $\eta$ both sides of (5.16) are analytic in $\sigma$. Hence we find that (5.16) holds for all values $\eta$ and $\sigma$ with

$$
-\operatorname{Im} \eta \in V_{+}, \quad-\operatorname{Im} \sigma \eta \in V_{+} .
$$


This allows defining the further continuation

$$
G_{j l}^{a b}(x, \eta)=\sum_{l^{\prime}=1}^{l} s_{j l l^{\prime}}(\sigma) G_{j^{\prime}}^{a b}\left(x, \frac{\eta}{\sigma}\right) \quad \text { if } \quad-\operatorname{Im} \frac{\eta}{\sigma} \in V_{+} .
$$

According to (5.12-5.14) the first operator of each subsequence satisfies a power scaling law

$$
C_{j 1}(x, \sigma \eta)=\sigma^{c_{j}} C_{j 1}(x, \eta) .
$$

We will show in general that every composite operator can be reduced to operators satisfying power scaling laws. To this end we introduce new operators $Q_{j k}^{a b}$ by

$$
Q_{(j)}^{a b}(x, \eta)=t_{j}(\eta) C_{(j)}^{a b}(x, \eta)
$$

where $t_{j}(\eta)$ denotes the matrix

$$
\begin{aligned}
t_{j}(\eta) & =\lim _{\varepsilon \rightarrow+0}\left(\sqrt{-\eta^{2}+i \varepsilon \eta_{0}}\right)^{c_{j}} s_{j}\left(\frac{1}{\sqrt{-\eta^{2}+i \varepsilon \eta_{0}}}\right) \\
& =\lim _{u \rightarrow+0}\left(\sqrt{-(\eta-i u)^{2}}\right)^{c_{j}} s_{j}\left(\frac{1}{\sqrt{-(\eta-i u)^{2}}}\right) .
\end{aligned}
$$

$\sqrt{-(\eta-i u)^{2}}$ is defined in $u \in V_{+}$by continuing from spacelike $\eta$ with $\sqrt{-\eta^{2}}>0$. According to Theorem 4 (5.20) defines an operator in $\mathscr{S}_{x \eta}^{\prime}\left(D_{0}\right)$. The scaling law of (5.20) immediately follows from (5.9) and (5.14)

$$
Q_{(j)}^{a b}(x, \sigma \eta)=\sigma^{c_{j}} Q_{(j)}^{a b}(x, \eta) .
$$

The operators $C_{j l}^{a b}$ can easily be expressed as linear combinations of the $Q_{j l}^{a b}$ :

$$
C_{(j)}^{a b}(x, \eta)=\lim _{\varepsilon \rightarrow+0}\left(\sqrt{-\eta^{2}+i \varepsilon \eta_{0}}\right)^{-c_{j}} s_{j}\left(\sqrt{-\eta^{2}+i \varepsilon \eta_{0}}\right) Q_{(j)}^{a b}(x, \eta) .
$$

Combining the power scaling law (5.22) with analyticity we will determine the general form of the operators $Q_{j l}^{a b}$ in the following section.

\section{Composite Operators with Power Scaling Law}

In the last section it was shown that composite operators either satisfy the power scaling law (5.19) in $\eta$ or can be written as linear combinations of such operators. We summarize the properties of these operators $Q_{j l}^{a b}$ which have been established so far. The subscripts $j, l$ will be omitted throughout this section. 
(i) $Q^{a b}(x, \eta)$ is an operator in $\mathscr{S}_{x \eta}^{\prime}\left(D_{0}\right)$.

(ii) The even and odd parts.

satisfy

$$
Q^{\text {even }}=Q^{12} \pm Q^{21}, \quad Q^{\text {odd }}=Q^{12} \mp Q^{21}
$$

$$
\begin{aligned}
& Q^{\text {even }}(x,-\eta)=Q^{\text {even }}(x, \eta) \\
& Q^{\text {odd }}(x,-\eta)=-Q^{\text {odd }}(x, \eta) .
\end{aligned}
$$

(iii) The matrix element

$$
g^{()}(x, \eta)=\left(\Phi, Q^{()}(x, \eta) \Psi\right) \quad \Phi, \Psi \in B
$$

satisfies the analytic properties of Theorem 3.

(iv) A power scaling law holds for the operator

$$
Q^{()}(x, \sigma \eta)=\sigma^{c} Q^{()}(x, \eta)
$$

and the continuations of the matrix element (6.3)

$$
\begin{array}{lrrr}
G^{()}(x, \sigma \eta)=\sigma^{c} G^{()}(x, \eta) & -\operatorname{Im} \eta \in V_{+}, & & \operatorname{Im} \sigma \eta \in V_{+} . \\
\hat{G}^{()}(x, \sigma \eta) & =\sigma^{c} \hat{G}(x, \eta) & \operatorname{Im} \eta \in V_{+}, & \operatorname{Im} \sigma \eta \in V_{+} .
\end{array}
$$

Using this information we will first investigate the $\eta$-dependence of the matrix elements (6.3).

\section{6a. Directional Dependence of Matrix Elements}

The following theorem states that the function $G^{a b}$ is a polynomial in $\eta$ apart from a power of $\eta^{2}$.

Theorem 5. The function $G^{a b}$ is of the general form

$$
G^{a b}(x, \eta)=\left(\sqrt{-\eta^{2}}\right)^{c} \Pi^{a b}\left(x, \frac{\eta}{\sqrt{-\eta^{2}}}\right)
$$

where $\Pi^{a b}(x, \zeta)$ is a polynomial in the components of the four vector $\zeta$.

For the proof we first list some properties of the function

$$
D_{f}^{()}(x, \eta)=\left(-\eta^{2}\right)^{f} G^{()}(x, \eta)
$$

which immediately follow from the corresponding properties of $G^{()}$. Here $f$ is a real number, $\left(-\eta^{2}\right)^{f}$ denotes the continuation of the positive values at spacelike $\eta$ into the regions $-\operatorname{Im} \eta \in V_{+}$and $\operatorname{Im} \eta \in V_{+}$.

(i) $D_{f}^{()}$is analytic and bounded by a polynomial in $-\operatorname{Im} \eta \in V_{+}$. 
(ii) The functions

$$
{\hat{D_{f}^{\text {ovd }}}}_{\text {oven }}^{\text {od }}(x, \eta)= \pm D_{f}^{\text {even }}(x,-\eta)=\left(-\eta^{2}\right)^{f} \hat{G}^{\text {oven }}(x, \eta)
$$

are analytic and bounded by a polynomial in $\operatorname{Im} \eta \in V_{+}$.

(iii) For spacelike $\eta$ the functions $D_{f}$ and $\hat{D}_{f}$ coincide

$$
D_{f}^{()}(x, \eta)=\hat{D}_{f}^{()}(x, \eta) \text { if } \eta \text { real and } \eta^{2}<0 .
$$

From property (i) we obtain (Theorem 2-8 of Ref. [2]) that $D_{f}^{()}$is a Laplace transform

$$
D_{f}^{()}\left(x, \eta_{1}-i \eta_{2}\right)=\frac{1}{(2 \pi)^{2}} \int e^{-i p\left(\eta_{1}-i \eta_{2}\right)} \tilde{d}_{f^{+}}^{()}(x, p) d p
$$

provided $\eta_{2} \in V_{+}$. From (ii) we find

$$
\hat{D}_{f}^{()}\left(x, \eta_{1}+i \eta_{2}\right)=\frac{1}{(2 \pi)^{2}} \int e^{-i p\left(\eta_{1}+i \eta_{2}\right)} \tilde{d}_{f^{-}}^{()}(x, p) d p
$$

with $\eta_{2} \in V_{+} . \tilde{d}_{f^{ \pm}}^{(\prime}(x, p)$ are tempered distributions in $\mathscr{S}^{\prime} p$ and have the support properties

$$
\begin{array}{lll}
\tilde{d}_{f^{+}}^{()}(x, p)=0 & \text { unless } & p \in \bar{V}_{+} \\
\tilde{d}_{f^{-}}(x, p)=0 & \text { unless } & p \in \bar{V}_{-} .
\end{array}
$$

According to Theorem 2-9 of Ref. [2] the limits of (6.10) and (6.11) for $\eta_{2} \rightarrow+0$ are

$$
\begin{aligned}
& \lim _{\eta_{2} \rightarrow+0} D_{f}^{()}\left(x, \eta_{1}-i \eta_{2}\right)=d_{f^{+}}^{()}\left(x, \eta_{1}\right) \\
= & \frac{1}{(2 \pi)^{2}} \int e^{-i p \eta_{1}} \tilde{d}_{f^{+}}^{()}(x, p) d p \text { in } \mathscr{S}_{\eta_{1}}^{\prime} \\
& \lim _{\eta_{2} \rightarrow+0} \hat{D}_{f}^{()}\left(x, \eta_{1}+i \eta_{2}\right)=d_{f^{-}}^{()}\left(x, \eta_{1}\right) \\
= & \frac{1}{(2 \pi)^{2}} \int e^{-i p \eta_{1}} \tilde{d}_{f^{-}}^{()}(x, p) d p \text { in } \mathscr{S}_{\eta_{1}}^{\prime} .
\end{aligned}
$$

Our aim is to choose $f$ such that $D_{f}^{()}=\hat{D}_{f}^{()}$for $\eta$ real and $\eta^{2} \neq 0$. To this end we form

$$
\begin{aligned}
H_{\mathrm{I}}(x, \eta) & =D_{-\frac{c}{2}}^{\text {even }}(x, \eta)=\frac{G^{\text {even }}(x, \eta)}{\left(\sqrt{-\eta^{2}}\right)^{c}} \\
H_{\mathrm{II}}(x, \eta) & =D_{-\frac{c+1}{2}}^{\text {odd }}(x, \eta)=\frac{G^{\text {odd }}(x, \eta)}{\left(\sqrt{-\eta^{2}}\right)^{c+1}} \\
\hat{H}_{\mathrm{I}}(\eta) & =H_{\mathrm{I}}(-\eta), \quad \hat{H}_{\mathrm{II}}(\eta)=-H_{\mathrm{II}}(-\eta)
\end{aligned}
$$


with the boundary values

$$
h_{\mathbf{I}^{ \pm}}=d_{-\frac{c}{2}, \pm}^{\text {even }}, \quad h_{\mathrm{II}, \pm}=d^{\text {odd }}, \frac{c+1}{2}, \pm .
$$

The scaling law then takes the form

$$
\begin{aligned}
H_{\mathrm{I}}(x, \sigma \eta) & =H_{\mathrm{I}}(x, \eta), \\
H_{\mathrm{II}}(x, \sigma \eta) & =\frac{1}{\sigma} H_{\mathrm{II}}(x, \eta) .
\end{aligned}
$$

(6.17) implies that $H_{\mathrm{I}}(\eta)$ is analytic for timelike $\eta$ and satisfies

$$
H_{\mathrm{I}}(x,-\eta)=H_{\mathrm{I}}(x, \eta) \quad \text { if } \quad \eta \in V_{ \pm} .
$$

For the proof we form

with

$$
H_{\mathrm{I}}(x, \sigma \eta)
$$

$$
\eta \in V_{+}, \quad \sigma=r e^{-i \varphi}, \quad r>0, \quad 0<\phi<\pi .
$$

Then $\sigma \eta$ lies in the regularity domain

$$
-\operatorname{Im}(\sigma \eta) \in V_{+} .
$$

Due to (6.17) the expression (6.20) is independent of $\sigma$ which shows that $H_{\mathrm{I}}$ is regular at $\eta \in V_{ \pm}$. Taking the limit $\phi \rightarrow 0$ and $\phi \rightarrow \pi$ of (6.20) we find (6.19). Combining (6.9) and (6.19) we get

$$
H_{\mathrm{I}}(x, \eta)=\hat{H}_{\mathrm{I}}(x, \eta) \text { if } \eta \text { real and } \eta^{2} \neq 0 .
$$

Similarly for $H_{\mathrm{II}}$

$$
\begin{gathered}
H_{\mathrm{II}}(x, \eta)=-H_{\mathrm{II}}(x, \eta) \text { if } \eta \in V_{1}, \\
H_{\mathrm{II}}(x, \eta)=\hat{H}_{\mathrm{II}}(x, \eta) \text { if } \eta \text { real and } \eta^{2} \neq 0 .
\end{gathered}
$$

As a consequence of $(6.21),(6.23)$ the boundary values from above and below agree for all real $\eta$ except $\eta^{2}=0$ :

$$
\begin{gathered}
\Delta_{\mathrm{I}}(x, \eta)=h_{\mathrm{I}+}(x, \eta)-h_{\mathrm{I}-}(x, \eta)=0 \\
\Delta_{\mathrm{II}}(x, \eta)=h_{\mathrm{II}+}(x, \eta)-h_{\mathrm{II}-}(x, \eta)=0
\end{gathered} \quad \text { if } \quad \eta^{2} \neq 0 .
$$

Now we use the following lemma which is proved in the appendix.

Lemma. Let $D(\eta) \in \gamma^{\prime}\left(R_{4}\right)$ be a distribution vanishing for $\eta^{2} \neq 0$. Then there exists an integer $k$ such that $\left(-\eta^{2}\right)^{k} D(\eta)=0$. If $D(\eta)$ is bounded in the norm \|\|$_{r s}$ any integer $k>|s|$ will do. 
As an immediate consequence we have, finally

$$
\begin{aligned}
\left(-\eta^{2}\right)^{m_{\mathrm{I}}} \Delta_{\mathrm{I}}(x, \eta) & =0 \\
\left(-\eta^{2}\right)^{m_{\mathrm{II}}} \Delta_{\mathrm{II}}(x, \eta) & =0
\end{aligned}
$$

for some positive integers $m_{\mathrm{I}}, m_{\mathrm{II}}$.

We next form

$$
\begin{gathered}
E_{\mathrm{I}}(x, \eta)=\left(-\eta^{2}\right)^{m_{\mathrm{I}}} H_{\mathrm{I}}(x, \eta)=D_{m_{\mathrm{I}}-\frac{c}{2}}^{\text {even }}(x, \eta) \\
E_{\mathrm{II}}(x, \eta)=\left(-\eta^{2}\right)^{m_{\mathrm{II}}} H_{\mathrm{II}}(x, \eta)=D_{m_{\mathrm{II}}-\frac{c+1}{2}}^{\text {odd }}(x, \eta) .
\end{gathered}
$$

We denote the boundary values by

$$
e_{\mathrm{I}, \pm}=\underset{m_{\mathrm{I}}-\frac{c}{2}, \pm}{\text { even }}, \quad e_{\mathrm{II}, \pm}=d_{m_{\mathrm{II}}-\frac{c+1}{2}, \pm}^{\text {odd }} .
$$

From (6.25) it follows

Hence

$$
\begin{aligned}
e_{\mathrm{I}+}(\eta) & =e_{\mathrm{I}-}(\eta) \\
e_{\mathrm{II}+}(\eta) & =e_{\mathrm{II}-}(\eta) .
\end{aligned}
$$

$$
\tilde{e}_{()}^{+}(p)=\tilde{e}_{()}^{-}(p),
$$

where () stands for the subscript I or II. Since $e_{()}^{+}$has support in $\bar{V}_{+}$and $e_{()}^{-}$has support in $\bar{V}_{-}$we get

$$
\tilde{e}_{()}^{ \pm}(p)=0 \text { unless } \quad p=0 .
$$

Therefore, the Fourier transform $e_{()}(x, \eta)$ must be a polynomial in $\eta$. On the other hand the scaling law

implies

$$
\begin{aligned}
E_{\mathrm{I}}(x, \sigma \eta) & =\sigma^{2 m_{1}} E_{\mathrm{I}}(x, \eta) \\
E_{\mathrm{II}}(x, \sigma \eta) & =\sigma^{2 m_{\mathrm{II}}-1} E_{\mathrm{II}}(x, \eta)
\end{aligned}
$$

Thus

$$
\begin{aligned}
& E_{\mathrm{I}}(x, \eta)=\left(\sqrt{-\eta^{2}}\right)^{2 m_{\mathrm{I}}} E_{\mathrm{I}}\left(x, \frac{\eta}{\sqrt{-\eta^{2}}}\right) \\
& E_{\mathrm{II}}(x, \eta)=\left(\sqrt{-\eta^{2}}\right)^{2 m_{\mathrm{II}}} E_{\mathrm{II}}\left(x, \frac{\eta}{\sqrt{-\eta^{2}}}\right) .
\end{aligned}
$$

$$
\begin{aligned}
& H_{\mathrm{I}}(x, \eta)=E_{\mathrm{I}}\left(x, \frac{\eta}{\sqrt{-\eta^{2}}}\right) \\
& H_{\mathrm{II}}(x, \eta)=\frac{1}{\sqrt{-\eta^{2}}} E_{\mathrm{II}}\left(x, \frac{\eta}{\sqrt{-\eta^{2}}}\right)
\end{aligned}
$$


and

$$
\begin{aligned}
G^{\mathrm{even}}(x, \eta) & =\left(\sqrt{-\eta^{2}}\right)^{c} E_{\mathrm{I}}\left(x, \frac{\eta}{\sqrt{-\eta^{2}}}\right) \\
G^{\mathrm{odd}}(x, \eta) & =\left(\sqrt{-\eta^{2}}\right)^{c} E_{\mathrm{II}}\left(x, \frac{\eta}{\sqrt{-\eta^{2}}}\right) .
\end{aligned}
$$

This leads to Eq. (6.6) of Theorem 4 with

$$
\Pi^{12}=\frac{1}{2}\left(E_{\mathrm{I}} \pm E_{\mathrm{II}}\right), \quad \Pi^{21}=\frac{1}{2}\left(E_{\mathrm{I}} \mp E_{\mathrm{II}}\right) .
$$

6b. Directional Dependence of the Operator

In the last section we have seen that every matrix element

$$
\left(\Phi, Q^{a b}(x \eta) \Psi\right) \quad \Phi, \Psi \in B
$$

is a polynomial in $\frac{\eta}{\sqrt{-\eta^{2}}}$ apart from a factor $\left(\sqrt{-\eta^{2}}\right)^{c}$. We proceed to establish a similar property for the operator $Q^{a b}$. To do this we must verify that the degree of the polynomial $\Pi^{a b}$ is uniformly bounded for all $x$ and $\Phi, \Psi \in B$.

The proof depends essentially on the following theorem, which is proved in the article [8] by Simon (Th. 11) and in Ref. [9].

Theorem (uniform boundedness). Let $D(\alpha ; \eta)$ be a family of distributions in $\mathscr{S}(\eta)$ parameterized by $\alpha$, and such that for every testing function $u(\eta)$ there exists a real number $M(u)$ with

$$
\left|\int d \eta D(\alpha ; \eta) u(\eta)\right| \leqq M(u) .
$$

Then the family $D(\alpha ; \eta)$ is uniformly bounded:

$$
\left|\int d \eta D(\alpha ; \eta) u(\eta)\right| \leqq M\|u\|_{r, s}
$$

for some norm \|\|$_{r, s^{\prime}}$ finite number $M$, and every testing function $u(\eta)$.

In order to use this theorem we construct $\Delta$ as in the last section and indicate the dependence on $\Phi, \Psi, x$ explicitly

$$
\begin{aligned}
& \Delta_{\mathrm{I}}(x, \eta ; \Phi, \Psi)=\lim _{\varepsilon \rightarrow+0} \frac{1}{\left(\sqrt{-\eta^{2}+i \varepsilon \eta_{0}}\right)^{c}}\left(\Phi,\left(Q^{\text {even }}(x, \eta)-Q^{\text {even }}(x,-\eta)\right) \Psi\right) \\
& \Delta_{\mathrm{II}}(x, \eta ; \Phi, \Psi)=\lim _{\varepsilon \rightarrow+0} \frac{1}{\left(\sqrt{-\eta^{2}+i \varepsilon \eta_{0}}\right)^{c+1}}\left(\Phi,\left(Q^{\text {odd }}(x, \eta)+Q^{\text {odd }}(x,-\eta)\right) \Psi\right)
\end{aligned}
$$


consider $\Delta_{()}(x, \eta ; \Phi, \Psi)$ as a family of distributions parameterized by $\Phi$ for fixed $\Psi$, use the continuity in $\Phi$ (for fixed $\Psi$ and testing function $\left.u(x, \eta) \in \mathscr{S}_{x \eta}\right)$ to bound the family for fixed $u$, then appeal to the uniform boundedness theorem to obtain

$$
\frac{1}{1+\|\Phi\|} \int d x d \eta u(x, \eta) \Delta_{()}(x, \eta ; \Phi, \Psi) \leqq M_{()}(\Psi)\|u\|_{r(\Psi), S(\Psi)} .
$$

By the lemma of Section 6 a this implies (since $\Delta_{()}=0$ for $\eta^{2} \neq 0$ ) that

$$
\left(\eta^{2}\right)^{\left.m_{(}\right)(\Psi)} \Delta_{()}(x, \eta ; \Phi, \Psi)=0 .
$$

Finally, locality in $x$ is used to remove the $\Psi$-dependence of $m_{()}(\Psi)$ and so complete the task. The details are as follows.

For every $\Phi \in B$, and testing function $u(x, \eta) \in \mathscr{S}_{x \eta}$

$$
\int d \eta d x \Delta_{()}(x, \eta ; \Phi, \Psi) u(x, \eta) \quad \Psi \in B
$$

is an inner product $(\Phi, \tilde{\Psi})$ (because of Theorem 4 ) and is thus continuous (or equivalently, bounded) in $\Phi$ :

$$
\left|\int d \eta d x \Delta_{()}(x, \eta ; \Phi, \Psi) u(x, \eta)\right|<M(\Psi, u)\|\Phi\| ;
$$

thus by the uniform boundedness theorem (6.38) and (6.39) are established and it remains only to remove the $\Psi$-dependence of $m_{(}(\Psi)$. To resolve the last problem we choose the vacuum $\Omega$ for the vector $\Psi$. Then

implies

$$
\left(-\eta^{2}\right)^{\left.m_{(}\right)(\Omega)} \Delta_{()}(x, \eta ; \Phi, \Psi)=0
$$

$$
N_{()}(x, \eta) \Omega=0
$$

where $N_{()}$denotes one of the operators

$$
\begin{gathered}
N_{\mathrm{I}}(x, \eta)=\left(-\eta^{2}\right)^{m_{\mathrm{I}}-\frac{c}{2}}\left(C^{\mathrm{even}}(x, \eta)-C^{\mathrm{even}}(x,-\eta)\right) \\
N_{\mathrm{II}}(x, \eta)=\left(-\eta^{2}\right)^{m_{\mathrm{II}}-\frac{c+1}{2}}\left(C^{\mathrm{odd}}(x, \eta)+C^{\text {odd }}(x,-\eta)\right) \\
\left(\Phi, N_{()}(x, \eta) \Psi\right)=\left(-\eta^{2}\right)^{\left.m_{(}\right)(-\Omega)} \Delta_{()}(x, \eta ; \Phi, \Psi) .
\end{gathered}
$$

Since $N_{(}$is local in $x$ relative to the basic fields $O_{j}$

$$
\left[N_{()}(x, \eta) O_{j}(y)\right]_{ \pm}=0 \quad \text { on } \quad D_{0} \quad \text { if } \quad(x-y)^{2}<0
$$

the relation (6.41) necessarily implies [10]

Hence

$$
N_{()}(x, \eta)=0 \text {. }
$$

$$
\left(-\eta^{2}\right)^{\left.m_{(}\right)} \Delta_{()}(x, \eta ; \Phi, \Psi)=0 \quad \Phi, \Psi \in B
$$


where $m_{()}$is independent of $x, \Phi$ and $\Psi$. Thus the degree of $\Pi^{a b}$ in (6.6) is uniformly bounded for $x$ and $\Phi, \Psi \in B$

$$
\begin{gathered}
\left(\Phi, Q^{a b}(x, \eta) \Psi\right)=\lim _{\varepsilon \rightarrow+0}\left(\sqrt{-\eta^{2}+i \varepsilon \eta_{0}}\right)^{c} \Pi^{a b}(x, \zeta, \Phi, \Psi) \\
\zeta=\frac{\eta}{\sqrt{-\eta^{2}+i \varepsilon \eta_{0}}}, \Phi, \Psi \in B ; \quad \operatorname{degr} \Pi^{a b} \leqq N .
\end{gathered}
$$

Smearing out in $x$ and $\eta$ with a test function of $\mathscr{S}_{x \eta}$ and using continuity of the left hand side in $\Psi$ for $\Phi \in B$ given we can extend (6.45) to vectors $\Phi \in B, \Psi \in D_{0}$. Keeping $\Psi \in D_{0}$ fixed and using continuity in $\Phi$ we can further extend (6.45) to vectors $\Phi \in H, \Psi \in D_{0}$. It is then not difficult to verify that the coefficients of $H^{a b}$ represent matrix elements of field operators.

We summarize the results of this Section in

Theorem 6. The operator $Q_{j l}^{a b}$ has the general form

$$
\begin{aligned}
Q_{j l}^{a b}(x, \eta) & =\lim _{\varepsilon \rightarrow+0}\left(\sqrt{-\eta^{2}+i \varepsilon \eta_{0}}\right)^{c} \sum_{n=1}^{N} \sum_{\mu_{1} \ldots \mu_{n}} \zeta_{\mu_{1}} \ldots \zeta_{\mu_{n}} Q_{j l}^{a b \mu_{1} \ldots \mu_{n}}(x) \\
\zeta & =\frac{\eta}{\sqrt{-\eta^{2}+i \varepsilon \eta_{0}}} .
\end{aligned}
$$

The coefficient $Q_{a b}^{\mu_{1} \ldots \mu_{n}}(x)$ is an operator in $\mathscr{S}_{x}^{\prime}\left(D_{0}\right)$. Under inhomogeneous Lorentz transformations $Q_{j}^{a b \mu_{1} \ldots \mu_{n}}$ follows the usual transformation law of a tensor of rank $n$. The operators $Q_{j}^{a b \mu_{1} \ldots \mu_{n}}$ are local relative to each other and relative to the basic fields.

Combining this theorem with Eq. (5.23) we have as final result

Theorem 7. For $\eta^{2} \neq 0$ given, the composite operators $C_{j}(x, \eta)$ appearing in the Wilson expansion

$$
A_{a}(x+\varrho \eta) A_{b}(x-\varrho \eta)=\sum_{j=1}^{h} f_{j}(\varrho) C_{j}^{a b}(x \eta)+P_{k+1}(x, \eta, \varrho)
$$

are polynomials in the components of $\eta / \sqrt{-\eta^{2}}$. By a suitable equivalence transformation the Wilson expansion (6.47) can be brought into the form

$$
A_{a}(x+\varrho \eta) A_{b}(x-\varrho \eta)=\sum_{j=1}^{n} \sum_{l=1}^{N_{j}} f_{j l}(\varrho) C_{j l}^{a b}(x \eta)+R(x \eta \varrho)
$$

where the composite operators may be written as

$$
C_{(j)}^{a b}(x, \eta)=\lim _{\varepsilon \rightarrow+0}\left(\sqrt{-\eta^{2}+i \varepsilon \eta_{0}}\right)^{-c_{j}} s_{j}\left(\sqrt{-\eta^{2}+i \varepsilon \eta_{0}}\right) Q_{(j)}^{a b}(x, \eta) .
$$

The $N_{j} \times N_{j}$ matrix $s_{j}$ is given by (5.12). The general form of the operators $Q_{j l}^{a b}$ was stated in Theorem 6. 


\section{Appendix}

\section{I: Proof of the Lemma, Section 6a.}

Statement. Let $D(\eta)$ be a distribution in $\mathscr{S}^{\prime}\left(R^{4}\right)$, with $D(\eta)=0$ for $\eta^{2} \neq 0$, and with $\int d \eta D(\eta) u(\eta)<M\|u\|_{r s}$, for each testing function $u(\eta) \in \mathscr{S}\left(R^{4}\right)$. Then for each integer $k>|s|,\left(\eta^{2}\right)^{k} D(\eta)=0$.

Proof. \|\|$_{r s}$ denotes the norm $\|u\|_{r s}=\sum_{\substack{|\alpha|<r \\|\beta|<s}} \sup _{\eta}\left|\eta^{\alpha} d_{\eta}^{\beta} u\right|$. Let $v \in \mathscr{S}(R)$ be such that $v(y)=1$ for $|y|<\frac{1}{2}$ and $v(y)=0$ for $|y|>1$; since $D$ vanishes for $\eta^{2} \neq 0$, one has for real $b>0$ and each testing function $u$ that

$$
\int d \eta D(\eta)\left(\eta^{2}\right)^{k} u(\eta)=\int d \eta D(\eta)\left(\eta^{2}\right)^{k} u(\eta) v\left(\eta^{2} / b\right) .
$$

However, when $k>s$, we claim that given a real $\varepsilon>0$ one may pick $b>0$ such that $\left\|\left(\eta^{2}\right)^{k} v\left(\eta^{2} / b\right) u(\eta)\right\|_{r s}<\varepsilon / M$; thus the right hand side of (A.1) vanishes and the statement is proved. The claim may be verified as follows: $\left\|\left(\eta^{2}\right)^{k} u(\eta) v\left(\eta^{2} / b\right)\right\|_{r s}$ is a sum of terms of the form

$$
\begin{gathered}
\sup _{\eta}\left|\left(\eta^{l} d_{\eta}^{m} u(\eta)\right)\left(\left(\eta^{2}\right)^{k-k^{\prime}} g^{\left(k^{\prime \prime}\right)}\left(\eta^{2} / b\right)\right)\right| b^{-k^{\prime \prime}} \\
\leqq\left(\sup _{\eta}\left|\left(\eta^{l} d_{\eta}^{m} u(\eta)\right)\right|\right)\left(\sup \left|\left(\eta^{2}\right)^{k-k^{\prime}} g^{\left(k^{\prime \prime}\right)}\left(\eta^{2} / b\right)\right|\right) b^{-k^{\prime \prime}} \quad k^{\prime}+k^{\prime \prime} \leqq s
\end{gathered}
$$

each of which may be made arbitrarily small

$$
\sup _{\eta} b^{-k^{\prime \prime}}\left|\left(\eta^{2}\right)^{k-k^{\prime}} g^{\left(k^{\prime \prime}\right)}\left(\eta^{2} / b\right)\right|=b^{k-\left(k^{\prime}+k^{\prime \prime}\right)} \sup _{t}\left|(t)^{k-k^{\prime}} g^{\left(k^{\prime \prime}\right)}(t)\right| .
$$

Corollary. Since every distribution is bounded in some norm \|\|$_{r s}$ it follows that for every $D(\eta) \in \mathscr{S}^{\prime}\left(R^{4}\right)$ vanishing for $\eta^{2} \neq 0$, there exists an integer $k$ with $\left(\eta^{2}\right)^{k} D(\eta)=0$.

This corollary is sufficient for the purpose of Section $6 \mathrm{a}$; Section $6 \mathrm{~b}$ strictly speaking requires the stronger result (which may be proved analogously):

Corollary. Let $D(x ; \eta)$ be a distribution in $\mathscr{S}^{\prime}\left(R_{8}\right)$ bounded in some norm \|\|$_{r s}$ and vanishing for $\eta^{2} \neq 0$; then $\left(\eta^{2}\right)^{k} D(x, \eta)=0$ for $k>s$.

\section{References}

1. Wilson, K., Zimmermann, W.: NYU Preprint 1971.

2. Streater, R., Wightman, A.: PCT, spin and statistics. New York: Benjamin 1964.

3. See, for instance, Ref. (2).

4. Jost, R., Lehmann, H.: Nuovo Cimento 5, 1598 (1957). Dyson, F.: Phys. Rev. 110, 1460 (1958).

5. Zimmermann, W.: 1970 Brandeis Lectures Vol. I. Cambridge: MIT Press 1971, and preprint in preparation.

6. See, for instance, Ref. [2]. 
7. Boerner,H.: Representations of groups. New York: Amer. Elsevier Publ. Co. Inc. 1970.

8. Simon, B.: J. Math. Phys. 12, 1 (1971).

9. Gelfand,I.M., Shilov, G.E.: Generalized functions, Vol. II. Néw York: Academic Press 1968.

10. Schroer, B.: Unpublished.

Jost, R.: In: Caianiello, E. (Ed.): Lectures on field theory and the many-body problem. New York: Academic Press 1961.

Federbush, P., Johnson, K.: Phys. Rev. 120, 1926 (1960).

\author{
W. Zimmermann \\ Paul Otterson \\ Department of Physics \\ New York University \\ 251 Mercer Street \\ New York, N.Y. 10012, USA
}

